\title{
MONOTONE AND OPEN WHITNEY MAPS
}

\author{
ALEJANDRO ILLANES
}

\begin{abstract}
In this paper we prove that if $X$ is a locally connected continuum, then there exists a monotone and open Whitney map for $2^{X}$.
\end{abstract}

1. Introduction. A continuum is a nonempty compact connected metric space. A Peano continuum is a locally connected continuum. The hyperspaces of a continuum $X$ are the spaces $2^{X}=\{A \subset X: A$ is nonempty and compact $\}$ and $C(X)=\left\{A \in 2^{X}: A\right.$ is connected $\}$ metrized with the Hausdorff metric. A Whitney map for the hyperspace $\sharp$ of $X$ is a continuous function $\omega: \forall \rightarrow[0, \infty)$ such that $\omega(\{x\})=0$ for each $x \in X$, and if $A, B \in \not{A}$ and $A \subset B \neq A$, then $\omega(A)<\omega(B)$ is monotone if $\omega^{-1}(t)$ is connected for every $t \in[0, \infty)$.

It is known that every Whitney map for $C(X)$ is monotone and open [3, p. 1032]. In [6] it was shown that even when $X$ is a circle or an arc, Whitney maps for $2^{X}$ can be constructed which are neither monotone nor open. S. B. Nadler, Jr. has asked in $[6,14.63$ and 14.64] whether for every continuum $X$ there exists a monotone (or an open) Whitney map for $2^{X}$. Recently, W. J. Charatonik [2] answered both questions negatively by showing an example of a continuum $X$ in the plane which has neither monotone nor open Whitney maps for $2^{X}$. In this paper, we prove that if $X$ is a Peano continuum, then a monotone open Whitney map can be constructed for $2^{X}$.

Throughout this paper $X$ will denote a continuum with a metric $d$. A metric $\rho$ for $X$ is said to be convex provided that, given $x, y \in X$, there exists $z \in X$ such that $\rho(x, z)=\rho(x, y) / 2=\rho(y, z)$. It is easy to prove that if $\rho$ is a convex metric for $X$, then, for any $x, y \in X$, there exists a isometry $\sigma:[0, \rho(x, y)] \rightarrow X$ such that $\sigma(0)=x$ and $\sigma(\rho(x, y))=y$. X is said to admit a convex metric $\rho$ provided that $\rho$ is a convex metric for $X$ and the (original) topology on $X$ is the same as the topology for $X$ obtained by $\rho$. If $X$ admits a convex metric $\rho$, then $X$ is a Peano continuum [4]. The converse was proved independently by R. H. Bing [1] and E. E. Moise [5].

2. A monotone open Whitney map. Given a positive integer $n$ and $A \in 2^{X}$, we define $\omega_{n}(A)=\inf \left\{\varepsilon>0:\right.$ there exist $x_{1}, \ldots, x_{n} \in X$ such that $A \subset B_{\varepsilon}\left(x_{1}\right) \cup$ $\left.\cdots \cup B_{\varepsilon}\left(x_{n}\right)\right\}$, where $B_{\varepsilon}(x)=\{y \in X: d(x, y)<\varepsilon\}$. Then $\omega_{n}: 2^{X} \rightarrow[0, \infty)$ is continuous, $\omega_{n}(\{x\})=0$ for each $x \in X$ and $\omega_{n}(A) \leq$ diameter of $X$. So, if we define $\omega: 2^{X} \rightarrow[0, \infty)$ by $\omega(A)=\sum \omega_{n}(A) / 2^{n}$, then $\omega$ is continuous and $\omega(\{x\})=0$ for each $x \in X$.

To prove that $\omega$ is a Whitney map, we take $A, B \in 2^{X}$ such that $A \subset B \neq A$. Let $b$ be a point of $B-A$ and let $\varepsilon>0$ such that $B_{2 \varepsilon}(b) \cap A=\varnothing$. Let $m$ be

Received by the editors July 10, 1985 and, in revised form, October 2, 1985.

1980 Mathematics Subject Classification (1985 Revision). Primary 54B20, 54F25.

Key words and phrases. Continuum, hyperspace, Whitney map. 
the minimum positive integer for which there exist points $x_{1}, \ldots, x_{m}$ of $X$ such that $A \subset B_{\varepsilon}\left(x_{1}\right) \cup \cdots \cup B_{\varepsilon}\left(x_{m}\right)$. Then there exists $\alpha>0$ such that $\alpha<\varepsilon$ and $A \subset B_{\alpha}\left(x_{1}\right) \cup \cdots \cup B_{\alpha}\left(x_{m}\right)$. So $\omega_{m}(A) \leq \alpha<\varepsilon$. The choice of $\varepsilon$ and $m$ implies that there are no points $y_{1}, \ldots, y_{m} \in X$ such that $B \subset B_{\varepsilon}\left(y_{1}\right) \cup \cdots \cup B_{\varepsilon}\left(y_{m}\right)$. Then $\omega_{m}(B) \geq \varepsilon>\omega_{m}(A)$. This implies that $\omega(B)>\omega(A)$. Hence $\omega$ is a Whitney map.

THEOREM. If $d$ is a convex metric for $X$, then the Whitney map defined above is monotone and open.

Proof. Given a finite subset $F=\left\{p_{1}, \ldots, p_{n}\right\}$ of $X, x_{0} \in X$ and $\beta>0$ such that $F \subset B_{\beta}\left(x_{0}\right)$, we put $t_{1}=d\left(x_{0}, p_{1}\right), \ldots, t_{n}=d\left(x_{0}, p_{n}\right)$ and we choose isometries $\sigma_{i}:\left[0, t_{i}\right] \rightarrow X$ such that $\sigma_{i}(0)=x_{0}$ and $\sigma_{i}\left(t_{i}\right)=p_{i}$. We define a path $F:[0,1] \rightarrow 2^{X}$ by $F(t)=\left\{\sigma_{1}\left(t t_{1}\right), \ldots, \sigma_{n}\left(t t_{n}\right)\right\}$. Then, if $t \in[0,1], F(t) \subset B_{t \beta}\left(x_{0}\right)$, so that $\omega_{1}(F(t)) \leq t \beta$. It is easy to see that $\omega_{m}(F(t)) \leq \omega_{m}(F)+(1-t) \beta$ for each $m$, and that for each $m \geq n, \omega_{m}(F(t))=0=\omega_{m}(F)$. This implies that $\omega(F(t)) \leq t \beta / 2+\omega(F)-\omega_{1}(F) / 2+(1-t) \beta / 2-(1-t) \beta / 2^{n-1}=\omega(F)-\omega_{1}(F) / 2+$ $\beta / 2-(1-t) \beta / 2^{n-1}$.

First, we will prove that $\omega$ is open. By Theorem 14.65 of $[6]$, it is enough to prove that if we take a finite nondegenerate subset $F$ of $X$ and if we take an open subset $U$ of $2^{X}$ such that $F \in U$, then $\omega(F)$ is an interior point of $\omega(U)$. Choose $0<\varepsilon<1$ such that $\left\{A \in 2^{X}: H(A, F)<\varepsilon\right.$ (diameter of $\left.\left.X\right)\right\} \subset U$, where $H$ is the Hausdorff metric for $2^{X}$. We take $\beta<\omega_{1}(F) /\left(1-\varepsilon / 2^{n-1}\right)$ and $x_{0} \in X$ such that $F \subset B_{\beta}\left(x_{0}\right)$. Let us consider $F:[0,1] \rightarrow 2^{X}$ constructed as above. Then $\omega(F(1-\varepsilon / 2)) \leq \omega(F)-\omega_{1}(F) / 2+\beta / 2-\varepsilon \beta / 2^{n}<\omega(F)$. Moreover, given $t \in[1-\varepsilon / 2,1], H(F(t), F)<\varepsilon$ (diameter of $X)$, so that $F([1-\varepsilon / 2,1]) \subset U$.

It follows that there exists $\alpha>0$ such that $(\omega(F)-\alpha, \omega(F)] \subset \omega(U)$. Now, we consider the continuum $M=\mathrm{Cl}_{X}\left(B_{\varepsilon / 2}\left(p_{1}\right)\right)$. Then the set $M_{1}=\{F \cup\{x\}: x \in M\}$ is connected, $M_{1} \neq\{F\}, \omega(A) \geq \omega(F)$ for each $A \in M_{1}$ and $M_{1} \subset U$. Then there exists $\lambda>0$ such that the interval $[\omega(F), \omega(F)+\lambda)$ is contained in $\omega(U)$. Hence $\omega(F)$ is an interior point of $\omega(U)$. This proves that $\omega$ is open.

Now, we will prove that $\omega$ is monotone (compare with 14.66 of [6]). First, we will show that $\omega^{-1}([0, t))$ is connected for any $t \in \mathbf{R}, t>0$. Since $\mathcal{E}=\{F \in$ $2^{X}: \omega(F)<t$ and $F$ is finite $\}$ is a dense subset of $\omega^{-1}(([0, t))$, it will be enough to prove that $\mathcal{E}$ is connected. Take $F \in \mathcal{E}$. Let $\beta<\omega_{1}(F)+2(t-\omega(F))$ and $x_{0} \in X$ be such that $F \subset B_{\beta}\left(x_{0}\right)$. Then if we consider $F:[0,1] \rightarrow 2^{X}$ as above, $\omega(F(s))<t$ for each $s \in[0,1]$. Moreover, $F(0) \in F_{1}(X)\left(=\left\{\{x\} \in 2^{X}: x \in X\right\}\right), F_{1}(X) \subset \mathcal{E}$ and $F_{1}(X)$ is connected. This implies that $\mathcal{E}$ is connected. Hence $\omega^{-1}([0, t))$ is connected for each $t \in \mathbf{R}$. It follows that $\omega^{-1}([0, t])$ is connected for each $t \in \mathbf{R}$.

Since $2^{X}$ is unicoherent $[6,1.176]$, to prove that $\omega^{-1}(t)$ is connected, it is enough to prove that $\omega^{-1}([t, \infty))$ is connected. Take $t \in \mathbf{R}$, and $A \in \omega^{-1}([t, \infty))$. Notice that $\left\{A \cup\{x\} \in 2^{X} ; x \in X\right\}$ is a connected subset of $\omega^{-1}([t, \infty))$. Furthermore, $\left\{A \cup F \in 2^{X}: F\right.$ is a finite subset of $\left.X\right\}$ is a connected subset of $\omega^{-1}([t, \infty))$ containing $X$ in its closure. This implies that $\omega^{-1}([t, \infty))$ is connected. Therefore, $\omega^{-1}(t)$ is connected, and $\omega$ is monotone.

Corollary. If $X$ is a Peano continuum, then there exists a monotone open Whitney map for $2^{X}$. 


\section{BIBLIOGRAPHY}

1. R. H. Bing, Partitioning a set, Bull. Amer. Math. Soc. 55 (1949), 1101-1110.

2. W. J. Charatonik, A continuum $X$ which has no confluent Whitney maps, Proc. Amer. Math. Soc. 92 (1984), 313-314.

3. C. Eberhart and S. B. Nadler, Jr., The dimension of certain hyperspaces, Bull. Acad. Polon. Sci. Ser. Sci. Math. 19 (1971), 1027-1034.

4. K. Menger, Untersuchungen über allgemeine Matrik, Math. Ann. 100 (1928), 75-163.

5. E. E. Moise, Grille decomposition and convexification theorems for compact locally connected continua, Bull. Amer. Math. Soc. 55 (1949), 1111-1121.

6. S. B. Nadler, Jr., Hyperspaces of sets, Marcel Dekker, New York and Basel, 1978.

Instituto de Matematicas, Universidad Nacional Autonoma de Mexico, CirCuito Exterior, Ciudad Universitaria, Mexico 20, D. F. 